\title{
GALACTIC DYNAMICS AND DARK MATTER WITH SUB-MILLIARCSECOND ASTROMETRY
}

\author{
D. PFENNIGER \\ Geneva Observatory \\ University of Geneva \\ $\mathrm{CH}-1290$ Sauverny \\ Switzerland
}

\begin{abstract}
Improving the astrometric accuracy by one or two orders of magnitude over ground-based techniques will not only change our raw knowledge about the Galaxy, but it will also modify 1) the fundamental questions that can be addressed, and 2) the stellar dynamical concepts used so far. More detail in Galactic structure, such as the shape and flow in its putative bar, will be accessible. Also, with the instruments of the next generation the large scale dark matter distribution in the Galaxy, whether distributed in a spheroidal smooth halo or a massive outer disc made of cold clumpy gas, will be measurable. Techniques used for mapping the cosmic flow and mass distribution at Mpc scales and more might be applied to the solar neighbourhood to find the degree of clumpiness of the local matter distribution.
\end{abstract}

\section{Galaxies in an Astrometric Context}

The improvement by a factor $\sim 10$ on the accuracy of stellar astrometric measurements by the Hipparcos satellite over ground-based observations, and by a possible further factor $\sim 50$ down to perhaps $20 \mu$ as by future astrometric satellites ${ }^{[1]}$ will have profound consequences on the way we describe the Galaxy, and consequently other galaxies. Indeed many theoretical concepts or models presently operative in stellar dynamics, such as the concept of isolating integrals, need a certain degree of symmetry in the stellar distribution (such as time-invariance or axisymmetry) in order to be useful in practice.

The age-velocity dispersion relation and the vertex deviation were already features incompatible with a strictly time-independent or axisymmetric Galaxy. We can expect that such discrepancies with first order Galactic models will turn up more often with the publication of the Hipparcos measurements, and all the more so with the results of even more accurate instruments, since the accuracy level will increasingly exceed the level at which symmetric galaxy models are relevant.

While better data is coming, in galactic studies we can notice a slow but radical theoretical shift in the way galaxies are perceived, as sketched below. The observational objectives that have been considered as important in the past, such as the precise determination of the scale-lengths, or Oort's constants of axisymmetric and stationary Galaxy models, will become irrelevant for more precise models. 


\section{Galactic Dynamics at Large and Small Scales, Spiral Evolution}

In fact the detailed dynamical modeling of galaxies, which is increasingly performed by $N$-body simulations, shows a consistent but quite opposite picture than theoreticians had in mind for years. A disc galaxy can no longer be viewed as a stationary object over several $\mathrm{Gyr}^{[2]}$, that would form rapidly in an early phase and then would remain frozen for tens of rotational periods. On the contrary, disc galaxies seem to form progressively over Gyrs by a continuous accretion of gas at the disc periphery (e.g. [3]), and are sometimes subject to faster episodes of tidal or merger interactions with dwarfs or other galaxies. All the evidences we have by probing the Universe at cosmological distances is that galaxies did significantly evolve during the last 10 Gyr (see e.g. [4]). The morphology-radius relationship ${ }^{[5]}$ in galaxy clusters is particularly suggestive that spirals in such dense environments are destroyed by crossing a cluster only once. Contrary to elliptical galaxies, spiral galaxies should be viewed as relatively flexible, easily perturbed objects.

To understand the structure and evolution of an isolated disc galaxy one needs to include the gas dissipative component, that has to be efficient anyway to produce initially a condensed and flat disc. An energy dissipative disc tends to increase the fraction of rotational energy to pressure energy, because, contrary to energy, angular momentum is hardly dissipated. Ineluctably the disc reaches the Safronov-Toomre gravitational instability threshold well before every bit of matter is settled into circular motion. A striking property of the observed disc galaxies is that when they are close to this instability threshold, they are only marginally stable ${ }^{[6]}$. Marginal stability means that the dissipative factors are still acting now, and that their effect is counterbalanced by the global heating of the gravitational instability.

The main visible effect of such a marginal stability is to make galactic discs highly responsive to dynamical perturbations: such discs are strong amplifiers of the effect of a perturbation. In fact, most disc galaxies are developing instabilities producing spiral arms or stellar bars most of the time, hence the "spiral" name. The most massive and persistent structures resulting from large scale instabilities are the bars, which redistribute angular momentum and mass efficiently by gravitational torque in a few rotational periods only. As shown by $N$-body simulations ${ }^{[7],[8]}$, the final mass distribution of barred galaxy models adopts an exponential profile, precisely observed in the optical part of disc galaxies. Also, it is now clear that most disc galaxies display a substantial degree of non-axisymmetry such as bars and ovals in the central parts, and asymmetric $\mathrm{HI}$ discs and warps in the outer parts.

So, the old question to understand the persistence of spiral arms becomes irrelevant if spiral arms in fact grow, wind up, and fade away in a recurrent way. The typical time-scales of these fluctuations is the dynamical time, $10^{8}-10^{9} \mathrm{yr}$.

A central feature of spirals is the bulge, that for a long time was seen as an old stellar population component. But the large dispersion in abundances, metal-rich stars with large motions out of the plane ${ }^{[0]}$, and bluish bulges make it difficult to maintain the scenario that bulges formed rapidly in an early phase. Also, the absence of bulges in late-type galaxies should be understood. As an alternative, dynamical studies show that bulges may grow progressively as a result of the above mentioned instability episodes. Bars may dissolve into bulges by various mechanisms, such as gas accretion or galaxy satellite merging inside the bar ${ }^{[10],[2]}$. 
Another predicted feature associated with bars is their tendency to take a peanut or box shape when the bar is seen edge-on ${ }^{[1]}$. So the distinction between a bar and a bulge is artificial from the point of view of the dynamics. This property of stellar bars will be testable in the Galaxy, because its bulge does display a boxy shape, particularly in the near-infrared.

The global picture that emerges is that spirals may evolve along the Hubble sequence over Gyrs, conformably to the existing irreversible processes: general energy dissipation, bulge formation, star formation, and nucleo-synthesis. Spirals begin as late-type, asymmetric, small, bulgeless, metal-poor but gas-rich galaxies. During this secular evolution, the disc is symmetrized by more rotations, mass accretes at the warped disc periphery, the density and rotation velocity increase by the general dissipation, the bulge grows by internal instabilities, star formation increases the stellar mass and consumes the gas, and stellar evolution enriches the ISM gas. Faster phases of evolution with possible starbursts occur when galaxy satellites merge. Mergers between equal-sized galaxies produce elliptical galaxies by a rapid dynamical mixing of discs. Smaller mergers (satellite mass $<10 \%$ primary galaxy mass) produce big S0 bulges by heating the disc to large heights ${ }^{[2]}$.

\section{Dark Matter in Disc Galaxies: Cold Molecular Hydrogen?}

Then, in this evolution scenario one should also understand why the $M / L$ ratio decreases systematically along the Hubble sequence: most $(\sim 99 \%)$ of the mass in Sd galaxies is "dark", while most $(\gtrsim 50 \%)$ of the mass in Sa galaxies shines as a "normal" stellar population. A consistent proposition ${ }^{[12]}$ is that during the process of galaxy evolution (which is dynamically unavoidable whatever dark matter is made of) most of dark matter has been transformed into stars.

Therefore, not only dark matter should be mostly in some form of hydrogen, but furthermore this hydrogen should be in a sufficiently diluted phase in order to make stars later on. This excludes Jupiters and brown dwarfs. In this picture dark compact objects can form at most the fraction of dark matter that might exist in late type spirals, a minor part of the initial dark matter in early type galaxies.

In a critical investigation on the way gas mass is detected in the ISM (mainly by $\mathrm{HI}$ and $\mathrm{CO}$ ), our conclusion ${ }^{[13]}$ is that most gas may be invisible with present techniques if it is molecular hydrogen (the lowest energy state of hydrogen) at a temperature near the coldest cosmic temperature of $3 \mathrm{~K}$. This gas would be metal poor, in a highly clumpy state, along a fractal mass distribution that would extend at small scale, down to a few tens of AU, the already observed fractal cold $(5-100 \mathrm{~K})$ gas already observed in molecular clouds (in HI, CO and FIR) at scales in the range of $\sim 0.01-100 \mathrm{pc}$. Most of this gas would be located in the HI outer galactic discs, where neutral hydrogen is known to be proportional (by a factor 10-30) to dark matter anyway. One can see that in such a fractal structure, with a fractal dimension $D \sim 1.7$, clumps collide frequently at every scale. Yet the estimated energy dissipation time-scale is very long, several $\mathrm{Gyr}^{[13]}$. Also with $D<2$ (less than a surface!) the sky covering factor of the smallest clumps is very small, so the fractal looks in average much more transparent to the background sources than it would if the gas would have a larger $D$, like the one of common gases. 


\section{Objectives for Future Uses of Astrometric Data}

So, theory offers plenty of justifications to look at the Galaxy with a much finer spatial resolution than available before. At the expected level of accuracy planned for future astrometric satellites, $\ll 0.1$ mas, the huge amount of data will permit to map directly the star distribution and the first velocity moments over most of the optical Galaxy. The problems will be then to describe all the asymmetries, beginning by its bar and its warp, and then smaller scale bumps, such as spiral arms, or even smaller mass irregularities. According to self-consistent bar models ${ }^{[14],[8]}$ the streamings in the stellar velocity field due to a Galactic bar are expected to reach tens of $\mathrm{km} / \mathrm{s}$ within the bar and still several $\mathrm{km} / \mathrm{s}$ beyond the solar radius.

Combining these kinematical data with dynamical assumptions will allow to map the total local matter distribution around the Sun by injecting the first velocity moments of stellar populations into the Jeans equations. Contrary to earlier models, no symmetries should be assumed. If the velocity tensor is only partly known, in principle a method similar to the POTENT method ${ }^{[15]}$ might be used. Currently this method yields the total mass distribution at $100 \mathrm{Mpc}$ scale, knowing the radial velocity and distances of thousand of galaxies. This will be even more useful at the $100-1000 \mathrm{pc}$ scale because the transverse velocities will be known. Then the local distribution of dark matter near the Sun could be determined. We expect that dark matter, if associated with cold gas, might be more abundant outside the several $\sim 100 \mathrm{pc}$ wide hot gas bubble we live in.

With a parallax accuracy $\lesssim 0.1$ mas it will be possible to map the matter distribution in the Galactic nearby halo. Then from the kinematics it should be possible to discriminate whether dark matter is distributed smoothly in a round or fat spheroid as classically assumed, or, as we propose, in an outwards thickening outer disc made of cold clumpy gas.

\section{REFERENCES}

[1] Lindegren L., et al.: 1993, in European Space Agency Mission Concepts, Tome 1

[2] Pfenniger D.: 1992, in Physics of Nearby Galaxies, XIIth Moriond Astrophysics Meeting, T.X. Thuan et al. (eds.), Editions Frontières, Gif-sur-Yvette, p. 519

[3] Evrard A.E.: 1992, ibid. [2], p. 375

[4] Durret F. (ed.): 1994, Clusters of Galaxies, XIVth Moriond Astrophysics Meeting, Editions Frontières, Gif-sur-Yvette, in press

[5] Whitmore B.C.:1992, ibid. [2], p. 425; and 1994, in [4]

[6] Kennicutt R.C.: 1989, ApJ 344, 685

[7] Hohl F.: 1971, ApJ 168, 343

[8] Pfenniger D., Friedli D.: 1991, A\&A 252, 75

[9] Rich R.M.: 1992, ibid. [2], p. 153

[10] Pfenniger D., Norman C.: 1990, ApJ 363, 391

[11] Combes F., Debbasch F., Friedli D., Pfenniger D.: 1990, A\&A 233, 82

[12] Pfenniger D., Combes F., Martinet L.: 1994, A\&A 285, 79

[13] Pfenniger D., Combes F.: 1994, A\&A 285, 94

[14] Pfenniger D.: 1984, A\&A 141, 171

[15] Deckel A., Bertschinger E., Faber S.M.: 1990, ApJ 364, 349 